\title{
Modelo Cosmológico Quântico de Einstein-Aether com Fluido Perfeito de Vácuo
}

\author{
F. G. Alvarenga ${ }^{1}$ \\ Universidade Federal do Espírito Santo, ES, Brasil \\ G. A. Monerat ${ }^{2}$, O. Godoni ${ }^{3}$ e E. V. C. Silva ${ }^{4}$, \\ Universidade do Estado do Rio de Janeiro, RJ, Brasil \\ G. de O. Neto ${ }^{5}$ \\ Universidade Federal de Juiz de Fora, MG, Brasil.

${ }^{1 \text { flavio.alvarenga@ufes. }}{ }^{4}$ evasquez@uerj.br ${ }^{5}$ gilneto@ufjf.br

\section{Resumo}

A Cosmologia Quântica é uma teoria de condições iniciais que permite descrever o nascimento do Universo sem a indesejábel singularidade inicial tipo big bang. Nesse sentido, propõe-se um modelo quântico de gravidade acoplada ao campo de éter, a chamada teoria de Einstein-Aether, com conteúdo material de fluido de vácuo. A trajetória correspondente ao fator de escala do Universo é obtida e interpretada segundo o esquema de de Broglie-Bohm.

\section{O Modelo}

$\mathrm{Na}$ teoria de Einstein-Aether [1, 2, 3], quantidades cinemáticas quadráticas de vetores unitários tipo-tempo são introduzidas na ação integral:

$$
\begin{gathered}
\mathscr{S}=\frac{1}{16 \pi G}\left[\int_{M} d^{4} x \sqrt{-g} R+2 \int_{\partial M} d^{3} x \sqrt{h} K\right] \\
+\frac{1}{16 \pi G} \int_{M} d^{4} x \sqrt{-g}\left[\mathscr{K}_{m n}^{a b} \nabla_{a} u^{m} \nabla_{b} u^{n}\right. \\
\left.+\lambda\left(u^{a} u_{a}-1\right)\right] .
\end{gathered}
$$

Acima $R$ é o escalar de curvatura, $K$ é o traço da curvatura extrínseca, $\lambda$ é um multiplicador de Lagrange que assegura a a unitariedade do campo de éter $u^{n}$, e $\mathscr{K}_{a b}^{m n}$ é é o tensor

$$
\begin{gathered}
\mathscr{K}_{a b}^{m n}=c_{1} g^{a b} g_{m n}+c_{2} \delta_{m}^{a} \delta_{n}^{b} \\
+c_{3} \delta_{n}^{a} \delta_{m}^{b}+c_{4} u^{a} u^{b} g_{m n},
\end{gathered}
$$

sendo $c_{1}, c_{2}, c_{3}$ e $c_{4}$ constantes de acoplamento entre os campos de éter e gravitacional. Os novos termos quebram a simetria de Lorentz, pela seleção de um referencial privilegiado em cada ponto do espaçotempo, mas preservando as equações de segunda ordem como na Relatividade Geral. 


\section{Blucher Proceedings XI Encontro Científico de Física Aplicada Blucher}

A constante $G$ em (1) é definida como

$$
G=G_{N} \sigma ; \sigma=\left(1-\frac{c_{1}+c_{4}}{2}\right) .
$$

Daqui pra frente assume-se $16 \pi G_{N}=1$.

A homogeneidade e isotropia do Universo é descrita pela métrica de Friedmann-Robertson-Walker com seção espacial plana,

$$
d s^{2}=-N(t)^{2} d t^{2}+a(t)\left(d r^{2}+r^{2} d \Omega\right),
$$

onde $N(t)$ é a função lapso, que corresponde à componente normal da quadrivelocidade, $a(t)$ é o fator de escala. A inserção da métrica reduz a ação (1) a forma

$$
S_{g}=\int d t\left(-6 \frac{\dot{a}^{2} a}{N \sigma}-\frac{6 \beta}{a N \sigma} \dot{a}^{2}\right)
$$

onde $\beta=c_{1}+3 c_{2}+c_{3}$.

A matéria é introduzida pelo chamado formalismo de Schutz [4], no qual a pressão de um fluido de vácuo $(p=-\rho)$ é expressa em termos de potencias de velocidade escalares $\phi, \theta$ e $S$ :

$$
U_{v}=\frac{1}{\mu}\left(\phi_{, v}+\theta S_{, v}\right)
$$

cada um satisfazendo sua própria equação de movimento. Acima $\mu$ é a entalpia específica, $S$ é a entropia específica. As variáveis $\phi$ e $\theta$ não têm significado físico claro.

A ação da matéria é especialmente simples:

$$
S_{f}=\int d^{4} x \sqrt{-g} p
$$

Relações termodinâmicas [5] permitem que a ação da matéria reduzida seja escrita como

$$
S_{m}=-\int d t \frac{a^{3} N}{\sigma} e^{S}
$$

Pelo formalismo canônico identificase a partir das Lagrangianas em (5) e (7) a super-Hamiltoniana do modelo Einsteinéter com fluido de vácuo:

$$
\mathscr{H}=-\frac{\sigma p_{a}{ }^{2}}{24 a\left(1+\frac{\beta}{a^{2}}\right)}+\frac{a^{3}}{\sigma} e^{S},
$$

a qual pode ser colocada numa forma mais sugestiva mediante a transformação canônica

$$
T=-p_{S} e^{-S} ; p_{T}=e^{S}
$$

resultando em

$$
\mathscr{H}=-\frac{\sigma p_{a}{ }^{2}}{24 a\left(1+\frac{\beta}{a^{2}}\right)}+a^{3} p_{T}
$$

\section{A Quantização}

É conveniente introduzir uma nova parametrização da função lapso, escrevendo-a como $N \frac{a}{\sigma}\left(1+\frac{\beta}{a^{2}}\right)$. A ação mantém a forma mas a superHamiltoniana é modificada para

$$
\mathscr{H}=-\frac{p_{a}^{2}}{24}+\frac{a^{4}}{\sigma}\left(1+\frac{\beta}{a^{2}}\right) p_{T} .
$$

O procedimento de quantização consiste na aplicação dos operadores $\hat{p}_{a} \rightarrow$ $-i \frac{\partial}{\partial a}$ e $\hat{p}_{T} \rightarrow-i \frac{\partial}{\partial T}$ em $(11)$ de modo a impor a equação de Wheeler-DeWitt, $\hat{\mathscr{H}} \Psi=0$, sobre a função de onda do Universo. Neste modelo, a equação assume a forma

$$
\frac{\partial^{2}}{\partial a^{2}} \Psi(a, \tau)+24 i \frac{\beta}{\sigma} a^{2} \frac{\partial}{\partial \tau} \Psi(a, \tau)=0
$$

Acima considerou-se $1+\frac{\beta}{a^{2}} \approx \frac{\beta}{a^{2}}$, uma vez que estamos interessados nos momentos iniciais do Universo. 


\section{Blucher Proceedings

A interpretação da variável $\tau=-T$ como o tempo cósmico $\left(N=\sigma / \beta a^{2}\right)$ e da equação (12) como uma genuína equação de Schrödinger, requer que o correspondente operador

$$
\hat{\mathscr{H}}=-\frac{\sigma}{24 \beta a^{2}} \frac{\partial^{2}}{\partial a^{2}}
$$

seja auto-adjunto. $\mathrm{O}$ fator de escala $a$ está restrito ao domínio $a>0$, tal que a quantização no minisuperespaço diz respeito somente as funções de onda definidas no semi-eixo $(0, \infty)$. Em tais circunstâncias deve-se impor certas condições de contorno sobre as funções de onda permitidas que garantam a autoadjuntice de (13). Assim sendo, o produto interno requerido é

$$
(\psi, \phi)=\int_{0}^{\infty} a^{2} \psi^{*}(a) \phi(a) d a
$$

com domínio do operador restrito àquelas funções de onda que obdeçam à condição

$$
\frac{\partial \Psi(a=0, \tau)}{\partial \tau}=0
$$

Propondo soluções estacionárias da equação (12), ou seja, soluções do tipo

$$
\Psi(a, \tau)=e^{-i E \tau} \psi(a),
$$

com $E$ sendo um parâmetro real, $\psi(a)$ obedece

$$
\frac{d^{2} \psi}{d a^{2}}+24 E \frac{\beta}{\sigma} a^{2} \psi=0 .
$$

Uma solução da equação (17) é

$$
\Psi_{E}(a)=\sqrt{a} C J_{1 / 4}\left(\sqrt{\frac{6 E \beta}{\sigma}} a^{2}\right),
$$

A norma é infinita, o que pode ser contornado pela construção de um pacote de ondas:

$$
\Psi(a, \tau)=\int_{0}^{\infty} C(E) e^{-i E \tau} \psi_{E}(a) .
$$

A adequada escolha de $C(E)$ permite a construção do pacote

$$
\Psi(a, \tau)=D a \frac{e^{\frac{-a^{4}}{4 M}}}{(2 M)^{\frac{5}{4}}},
$$

em que $M=g+\frac{i \sigma}{6 \beta} \tau$, com $g$ sendo uma constante arbitrária decorrente da escolha de $C(E)$.

\section{A Interpretação de Bohm-de Broglie}

Na interpretação de Bohm-de Broglie [6] um sistema físico individual compreende uma onda propagando-se no espaço e tempo juntamente com uma partícula que move-se continuamente guiada pela onda. A onda é matematicamente descrita por $\Psi(a, \tau)=R(a, \tau) e^{i S(a, \tau)}$, a qual é uma solução da equação (12).

A trajetória $a(\tau)$ é postulada por:

$$
p_{a}=\left.\frac{\partial S(a, \tau)}{\partial a}\right|_{a=a(\tau)},
$$

com o movimento dirigido pela equação de Hamilton-Jacobi:

$$
\frac{\partial S}{\partial \tau}+\frac{1}{24} a^{2} \frac{\sigma}{\beta}\left(\frac{\partial S}{\partial a}\right)^{2}+V+Q=0
$$

onde $Q$ é o chamado potencial quântico:

$$
Q=-\frac{1}{24 a^{2} R} \frac{\sigma}{\beta} \frac{\partial^{2} R}{\partial a^{2}} .
$$

Neste modelo sem termo de curvatura, o potencial clássico $V$ é nulo.

A amplitude da onda $R(a, \tau)$ e a fase $S(a, \tau)$ são identificadas em 20): 


\section{Blucher Proceedings XI Encontro Científico de Física Aplicada Blucher}

$$
\begin{gathered}
R(a, \tau)=\frac{D}{2^{5 / 4}\left(g^{2}+\frac{\sigma^{2} \tau^{2}}{36 \beta^{2}}\right)^{-\frac{5}{8}}} \\
. \exp -\frac{g a^{4}}{4\left(g^{2}+\frac{\sigma^{2} \tau^{2}}{36 \beta^{2}}\right)} ; \\
S(a, \tau)=\frac{a^{4} \sigma^{2} \tau^{2}}{24 \beta\left(g^{2}+\frac{\sigma^{2} \tau^{2}}{36 \beta^{2}}\right)} \\
-\frac{5}{2} \arctan \left(-\frac{\sigma \tau}{6 g \beta}\right) .
\end{gathered}
$$

A trajetória bohmiana $a(\tau)$ é obtida de $S(a, \tau)$ pela identificação de $\partial_{a} S$ com o momento canônico $\left|p_{a}\right|$ :

$$
12 \frac{\beta}{\sigma} \frac{\dot{a}}{a}=\frac{\sigma \tau}{6 \beta\left(g^{2}+\frac{\sigma^{2} \tau^{2}}{36 \beta^{2}}\right)}
$$

e portanto

$$
a(\tau)=a_{0}\left(g^{2}+\frac{\sigma^{2} \tau^{2}}{36 \beta^{2}}\right)^{4}
$$

onde $a_{0}$ é uma constante de integração arbitrária positiva. Esta solução é não singular, uma vez que o Universo tem um começo com tamanho finito em $\tau=0$.

O potencial quântico, calculado inserindo 24] em 23], é dado por:

$$
\begin{gathered}
Q(a, \tau)=-\frac{\sigma g}{96 \beta\left(g^{2}+\frac{\sigma^{2} \tau^{2}}{36 \beta^{2}}\right)^{2}}\left[\left(g^{2}+\frac{\sigma^{2} \tau^{2}}{36 \beta^{2}}\right)\right. \\
\left.-4 g a^{4}\right] .
\end{gathered}
$$

Ao potencial quântico está associada uma força

$$
F(a, \tau)=-\left.\frac{\partial Q(a, \tau)}{\partial a}\right|_{a=a(\tau)}=\frac{g a_{0}^{8} \sigma}{6 \beta a^{5}}
$$

a qual possui um caráter repulsivo atuando de modo a evitar a singularidade acerca da região $a=0$.

\section{Considerações Finais}

O modelo de Einstein-Aether que descreve a gravidade acoplada ao campo de éter ao ser quantizado com fluido-perfeito de vácuo revela um Universo não singular devido aos efeitos de um potencial quântico repulsivo. As figuras $1 \mathrm{e}$ 2 ilustram respectivamente o fator de escala e o potencial quântico em função do tempo. O potencial quântico atinge o máximo quanto o fator escala é mínimo, e a partir daí os efeitos quânticos decrescem e a tendência é que a evolução temporal siga o previsto pelo Modelo Cosmológico Padrão. Já está em fase de conclusão a generalização do modelo para um fluido barotrópico arbitrário e a comparação com os modelos sem campo de éter [7] com indicativos de mudança na potência em $\tau$ da dependência do fator de escala.

\section{Agradecimentos}

Os autores agradecem à comissão organizadora do XI Encontro Científico de Física Aplicada pela excelência do evento. G. A. Monerat agradece à FAPERJ pelo apoio financeiro (Proc. E26/010.101230/2018).

\section{Referências}

[1] M. Campista, R. Chan, M. F. A. Silva, O. Goldoni, V.H. Satheeshkumar e J. F. V. Rocha. Canadian Journal of Physics, vol. 98, n. 10 (2020). 


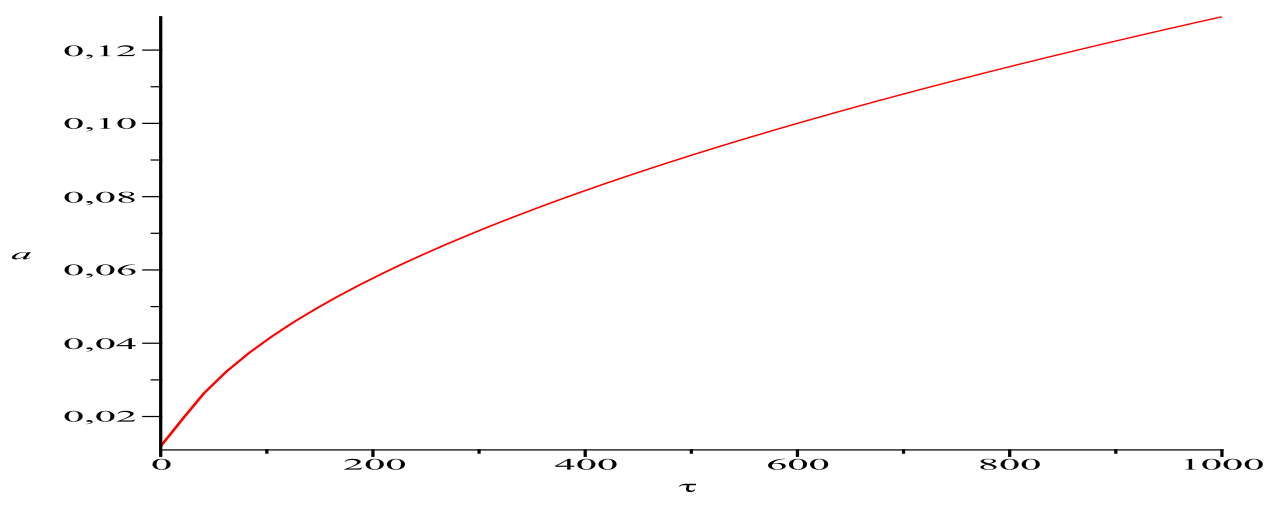

Figura 1: Fator de escala $a(\tau)$.

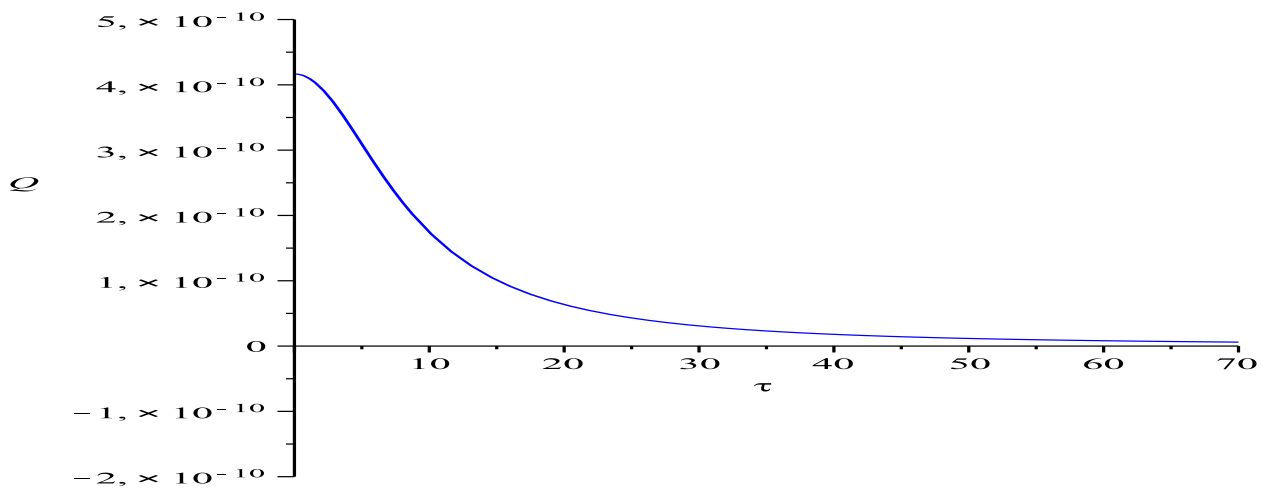

Figura 2: Potencial quântico $Q(\tau)$.

[2] N. Dimakis, T. Pailas, A. Paliathanasis, G. Leon, Petros A. Terzis e T. Christodoulakis Quantization of Einstein-aether Scalar field Cosmology. [arXiv:2008.00746v1 [gr-qc]].

[3] G. Leon, A. Paliathanasis e N. Dimakis. Exact Kantowski-Sachs spacetimes in Einstein-Aether Scalar field theory. [arXiv:2010.02775v2 [gr-qc]].

[4] F. G. Alvarenga, R. G. Furtado, R. Fracalossi e S. V. B. Gonçalves. Bra- zilian Journal of Physics, v. 47, p. 96 (2016).

[5] V. G. Lapchinskii e V. A. Rubakov. Theo. Math. Phys. 33. 1076 (1977).

[6] N. Pinto Neto. Teorias e Interpretações da Mecânica Quântica. São Paulo, Livraria da Física (2010).

[7] F. G. Alvarenga, J. C. Fabris, N. A. Lemos, G. A. Monerat, Gen. Rel. Grav. 34, 651 (2002). 